\title{
Research of Ambiguity Fast Resolution in GPS Orientation Determination
}

\author{
1st Chen Liang \\ College of Aerospace and Material Engineering, National \\ University of Defense Technology, \\ Changsha 410073, China
}

\begin{abstract}
Real-time GNSS orientation determination is valuable in many fields. In order to achieve high accuracy orientation with GNSS, the difference carrierphase observation must be employed. The carrier-phase measurements are affected by integer ambiguities, which must be resolved in order to take advantage of the high carrier-phase measurement precision. The information of the baseline length is exploited to reduce the correlation of ambiguities. So the size of integer ambiguities search space can be reduced. In order to make float ambiguity uncorrelated completely, float transformation matrix is constructed by Cholesky decomposition, so the speed of integer ambiguities searching can be improved. The experiment shows the method can improve the speed of integer ambiguities searching.
\end{abstract}

Keywords-Integer ambiguity, Decorrelation, Searching space, Fast orientation, Baseline length

\section{INTRODUCTION}

With the quick developing of GPS, high precision relative GPS positioning is used in more and more areas. Attitude determination with GPS signals has been deeply explored and attracted much attention in recent years. With the integer ambiguities have been resolved, the baseline solutions can be used to determine the orientation of the platform.The GPS orientation measurements presents advantages: it is driftless, it is virtually maintenance-free, and it does not need any ground reference. Jurgens employed the method to take the orientations of artilleries[1]. SPARTAN spacecraft took GPS to estimate its azimuth and pitching[2].

In order to obtain accurate measurements of a plan, GPS carrier phase observations must be employed. Application of GPS accurate orientation in aviation field needs the ability to resolve the integer values of the double difference carrier phase ambiguities quickly. Many methods were brought to resolve integer ambiguity in a lot of real time fields. The most famous method is the least-squares ambiguity decorrelation adjustment (LAMBDA) introduced by Teunissen. This method can calculate the ambiguity in a single epoch theoretically. But the numerical efficiency is still low when it is used in plane. To reduce the burden of the computer in plane, a new method of orientation is introduced in this contribution.

\author{
2nd Wu Jie \\ College of Aerospace and Material Engineering, National \\ University of Defense Technology, \\ Changsha 410073, China
}

The method introduced in this contribution with constraint of length of baseline is a modification of the LAMBDA. With the modified method the bias for float resolution can be reduced, so the ambiguity searching space can shrink. The computional complexity to calculate the transform matrix $\mathrm{Z}$ in LAMBDA method is high. The method introduced in this contribution needs not to calculate the transform matrix, so it is more efficient.

\section{ANALYSIS OF AMBIGUITY ESTIMATION}

Methods to calculate the ambiguity can be divided into three groups. They are static mode which needs a long time to collect data, exchanging antennas method and ambiguity searching method. Only the last one can be used in real time field. It needs only one epoch data to determine the integer ambiguities. So the method is called RTK (Real Time Kinematics) or OTF (On- The- Fly ). The typical integer ambiguity resolution technique has two steps. Firstly, calculate ambiguity in real number domain. Secondly, scan all integers around the real number solution by least square method. In these methods, it is difficult to set the size of the search space which should be big enough so the real integer ambiguity can be include in the search space and it should be small so the calculation of searching can be reduced.

Ambiguity searching methods can be divided into three groups by different observations. One searches integer ambiguity in measuring domain by the observation of code[3]. The precision depend on the precision of code measurement which is poor generally. So this group of mehods is often noneffective.

Another group is searching integer ambiguity in coordinate domain. Such as AFM (Ambiguity Function Method) which finds initial solution using code measurment and searches the real position in three-dimension space around the center of the initial solution. The advantage of this method is that it is roubust to cycle slip and the disadvantage is that it desires high precision of code measurment.

The third group is searching integer ambiguity in ambiguity domain which based on the theory of least square[4]. These methods have two steps. Firstly, an ordinary least-squares solution is computed. This solution is often referred to as the float solution. Then calculate the integer ambiguity. The solution of integer ambiguity must be obtained by means of searching. The float ambiguity variance matrix is non-diagonal. So the search space is highly elongated. The efficiency of the searching is poor. 
Many methods is referred to solute this question. For expampl, fast ambiguity resolution approach (FARA)[5], least-square ambiguity search technique (LSAST)[6], fast ambiguity search filter (FASF)[7], modified cholesky decomposition method[8], least-squares ambiguity decorrelation adjustment(LAMBDA) $[9,10]$, and so on. These methods can find the correct solution in most of the time. But the computing cost of these methods is high.

\section{ANALASYS OF LAMBDA}

Many of the existing techniques make good use of the popular widelaning technique which uses both L1 and L2 frequency measurement. The widelane phase observable, with its relatively long wavelength is considered an useful linear combination. Double-differenced (DD) phase data can achieve relatively small ionospheric delay, small satellites and recver clock noise. So the DD widelane linear combination is widely used. But DD phase observation has relatively high noise and observation vector is correlated, so it is not easy to confirm the integer ambiguity.

LAMBDA is an optimal technique to calculate the integer ambiguities. The float ambiguity is correlated, so the searching ambiguity is difficult. LAMBDA takes two steps to calculate the integer ambiguity. The first step is transformming the float ambiguity to another space, in which the transformed ambiguity vector has low correcation. The second step is searching the integer ambiguity in the transformed space.

We will consider the two-dimensional case. The search space which includes $a_{1}$ and $a_{2}$ is a ellopsoidal region. The ambiguities are not independent, so the search space is outstretched shown in figure 1.a. We do not know the shape of the region, so we must search the integer ambiguities in the rectangle region. The rectangle region which covers the ellopsoidal region is named enclosing box. So the number of candidates is very large. If the ambiguities are independent, the shape of searching is almost a roundness region shown in figure 1.b, the number of candidates can be reduced.
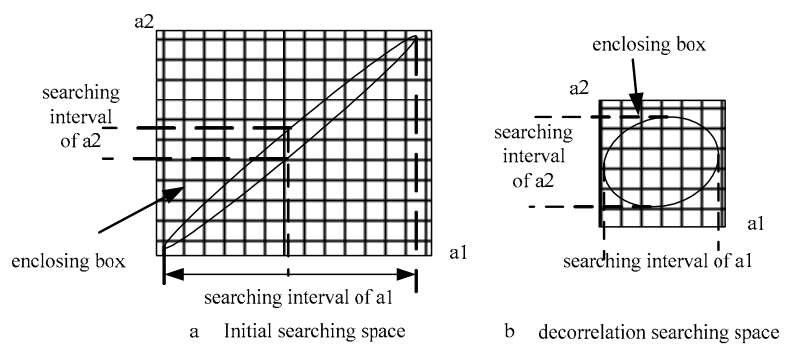

Figure 1 Ambiguity search space

$(\boldsymbol{a}-\hat{\boldsymbol{a}})^{T} \boldsymbol{Q}_{\hat{a}}^{-1}(\boldsymbol{a}-\hat{\boldsymbol{a}})$ follows $\chi^{2}$ distribution almostly. Where $\boldsymbol{a}$ is the original integer ambiguity vector, $\hat{\boldsymbol{a}}$ is the real value least-squares estimate which is called floating ambiguity, and $\boldsymbol{Q}_{\hat{\boldsymbol{a}}}$ is corresponding variance-covariance matrix. If the degree of confidence is given, a constant $\mathrm{C}$ can be confirmed. Then the integer ambiguities can be researched with the follow inequation.

$$
(\boldsymbol{a}-\hat{a})^{T} \boldsymbol{Q}_{\hat{a}}^{-1}(\boldsymbol{a}-\hat{\boldsymbol{a}})<\mathrm{C}
$$

The ambiguity search space is defined with inequalition (1). It is a set of vector $\boldsymbol{a}$ with the space is $\hat{\boldsymbol{a}}$. The shape of the space is governed by the variance-covariance matrix

$\boldsymbol{Q}_{\hat{a}}$ and the size is governed by C. The shape can be described by condition number which equal to the ratio of the maximal singular value and the minimal singular value of $\boldsymbol{Q}_{\hat{\boldsymbol{a}}}$. The bigger the condition number is the longer the search space will be.

We can transformat the ambiguity vector with Gauss method which read $\boldsymbol{z}=\boldsymbol{Z}^{\boldsymbol{T}} \boldsymbol{a}$. $\boldsymbol{z}$ is used to denote the transformed ambiguity vector which has low correlation. The variance-covariance matrix of $\hat{\boldsymbol{a}}$ reads $\boldsymbol{Q}_{z}=\boldsymbol{Z}^{T} \boldsymbol{Q}_{\hat{a}} \boldsymbol{Z}$. To keep the transformed problem to be equivalent to the original original problem, the matrix $\boldsymbol{Z}$ needs to have following properties[2]: (1) Elements of $\boldsymbol{Z}$ must be integer; (2) $\operatorname{Det}(\boldsymbol{Z})=1$.

$$
Z=\prod_{i=1}^{n-1} T_{L_{i}} T_{U_{i}}
$$

There is no normal method to obtain matrix $\boldsymbol{Z}$. With the developing requairment of real time positioning with GPS, many transformation methods are introducedlast few years. Transformation in LAMBDA uses upper triangular matrixes and lower triangular matrixes to obtain matrix $\boldsymbol{Z}$ with Gaussian transformation[6]. The method has perfect mathematical theory, but the process of disposing is complex.The transformation matrix can be written as

The transformed floating ambiguity can be written as $\hat{\boldsymbol{z}}=\boldsymbol{Z} \hat{\boldsymbol{a}}$. The variance-covariance matrix of $\hat{\boldsymbol{z}}$ reads $\boldsymbol{Q}_{\hat{z}}=\boldsymbol{Z} \boldsymbol{Q}_{\hat{a}} \boldsymbol{Z}^{T}$. (1) is equivalent to(3)

$$
\min (\hat{z}-z)^{T} \boldsymbol{Q}_{\hat{z}}^{-1}(\hat{z}-z), \quad z \in Z^{n}
$$

$\boldsymbol{Q}_{\hat{z}}$ should be a diagonal matrix in theory. But because of the integer restriction of the transformation matrix $\boldsymbol{Z}$, the correlation of the double difference ambiguities is difficult to eliminate. And the process to calculate matrix $\boldsymbol{Z}$ is complex, and the calculating efficiency is low.

The integer transform matrix $\boldsymbol{Z}$ can only reduce the correlation, but can not get rid of the correlation clearly. So $\boldsymbol{Q}_{\hat{z}}$ is close to a diagonal matrix, but not a diagonal matrix.

It is difficult to confirm the searching space of every dimension of vector $z$ directly. The searching space of vector $\boldsymbol{z}$ can be confirmed by a sequential method with following.

$$
\frac{\left(\hat{z}_{1}-z_{1}\right)^{2}}{\sigma_{\hat{z}_{1}}^{2}}+\cdot \cdot+\frac{\left(\hat{z}_{i \mid}-z_{i}\right)^{2}}{\sigma_{\hat{z}_{||}}^{2}}+\cdot \cdot+\frac{\left(\hat{z}_{\mid \mathrm{N}}-z_{n}^{2}\right)}{\sigma_{z_{i \mid N}}^{2}} \leq C
$$

Where $\hat{z}_{i \mid \mathbf{I}}$ denotes $\hat{z}_{i \mid(i-1, \cdots 1)}$ which is a conditional estimate for $z_{i}$ conditioned on $z_{j}$ with $j=1,2, \cdots i-1$. Searching space of every dimension of $\boldsymbol{z}$ is

$$
\left(\hat{z}_{i \mid \mathrm{I}}-z_{i}\right)^{2} \leq \sigma_{\hat{z}_{i \mid \mathrm{I}}}^{2}\left[C-\sum_{j=1}^{i-1} \frac{\left(\hat{z}_{j \mid \mathrm{J}}-z_{j}\right)^{2}}{\sigma_{\hat{z}_{j \mid \mathrm{J}}}^{2}}\right]
$$


Where $i=1,2, \cdots n-1$. Computional complexity of LAMBDA almost focuses on the obtainment of transformation matrix $\boldsymbol{Z}$. Because of the integer restriction, the transformation matrix $\boldsymbol{Z}$ can not be obtained by decomposing the variance-covariance matrix $\boldsymbol{Q}_{\hat{\boldsymbol{a}}}$. It needs many times of matrix decomposition, rounding and multiplication to obtain transformation matrix $\boldsymbol{Z}$. If we can obtain a matrix like transformation matrix $\boldsymbol{Z}$ by decomposing the variance-covariance matrix $\boldsymbol{Q}_{\hat{a}}$ only once, the computional complexity can be reduced.

\section{RESEARCH OF INTEGER AMBIGUITY SEARCHING}

\section{BASED ON CHOLESKY DECOMPOSING}

Double differenced carrier phase observation equation reads

$$
\varphi_{12}^{j k}=\frac{1}{\lambda}\left(\boldsymbol{r}_{\boldsymbol{I}}^{\boldsymbol{k} \boldsymbol{\theta}}-\boldsymbol{r}_{\boldsymbol{I}}^{j \boldsymbol{\theta}}\right) \cdot \boldsymbol{X}_{12}-N_{12}^{j k}+\varepsilon_{12}^{j k}
$$

$\boldsymbol{X}_{12}$ is the baseline vector. $\boldsymbol{r}_{1}^{\boldsymbol{k} \theta}$ and $\boldsymbol{r}_{1}^{j \boldsymbol{\theta}}$ are the unit vector of the line from antenna 1 to satellite $\mathrm{k}$ and $\mathrm{j}$. Code pseudorange observation equation reads.

$$
\rho_{12}^{j k}=\left(\boldsymbol{r}_{\boldsymbol{I}}^{\boldsymbol{k} \boldsymbol{\theta}}-\boldsymbol{r}_{\boldsymbol{1}}^{j \boldsymbol{\theta}}\right) \cdot \boldsymbol{X}_{\mathbf{1 2}}+\eta_{12}^{j k}
$$

The length of baseline restriction equation is $\left|\boldsymbol{X}_{12}\right|=l$. This equation is nonlinear equation which is often used as a part of the object function while searching integer ambiguities. The precision of the floating ambiguities can be increased if the length of baseline restriction can be used to calculate the floating ambiguities. So the value of elements of varianc-covariance matrix can be reduced and the correlation of the ambiguities can be reduced. The precision of floating ambiguities can be modified by linear expanding at the point of initial baseline vector.

The carrier phase and pseudorange observation equation

$$
\begin{gathered}
\varphi_{12}^{\mathrm{jk}}-\frac{1}{\lambda}\left(\boldsymbol{r}_{2}^{\boldsymbol{k} \boldsymbol{\theta}}-\boldsymbol{r}_{2}^{j \boldsymbol{\theta}}\right) \cdot \boldsymbol{X}_{12 \boldsymbol{\theta}}=\frac{1}{\lambda}\left(\boldsymbol{r}_{2}^{k \boldsymbol{\theta}}-\boldsymbol{r}_{2}^{j \boldsymbol{\theta}}\right) \cdot \boldsymbol{d} \boldsymbol{X}-N_{12}^{j k}+\varepsilon_{12}^{j k} \\
\rho_{12}^{j k}-\left(\boldsymbol{r}_{2}^{\boldsymbol{k} \boldsymbol{\theta}}-\boldsymbol{r}_{2}^{j \boldsymbol{\theta}}\right) \cdot \boldsymbol{X}_{12 \boldsymbol{\theta}}=\left(\boldsymbol{r}_{2}^{\boldsymbol{k} \boldsymbol{\theta}}-\boldsymbol{r}_{2}^{j \boldsymbol{j}}\right) \cdot \boldsymbol{d} \boldsymbol{X}+\eta_{12}^{j k} \\
\boldsymbol{r}_{2}^{\boldsymbol{k} \boldsymbol{\theta}} \text { and } \boldsymbol{r}_{2}^{j \boldsymbol{\theta}} \text { are are the unit vector of the line from }
\end{gathered}
$$

initial position of antenna 1 to satellite $\mathrm{k}$ and j. $\boldsymbol{X}_{120}$ is the initial baseline vector. $\boldsymbol{d} \boldsymbol{X}$ is the modification vector of $X_{120}$. The equation of baseline length reads

$$
l-\left|\boldsymbol{X}_{120}\right|=\frac{1}{\left|\boldsymbol{X}_{120}\right|} \boldsymbol{X}_{120} \bullet \boldsymbol{d} \boldsymbol{X}+\gamma_{12}
$$

$\boldsymbol{d} \boldsymbol{X}, \hat{\boldsymbol{a}}$ can be obtained from (8), (9), and (10). The variance-covariance matrix $\boldsymbol{Q}_{\hat{a}}$ is positive, it can be decomposed to a lower triangular matrix $\boldsymbol{L}$ by Cholesky method: $\boldsymbol{I}=\boldsymbol{L} \boldsymbol{Q}_{\hat{a}} \boldsymbol{L}^{\boldsymbol{T}} . \hat{\boldsymbol{a}}$ is transforme as:

$$
\hat{\boldsymbol{a}}^{\prime}=L \cdot \hat{\boldsymbol{a}}
$$

Elements of vector $\hat{\boldsymbol{a}}^{\prime}$ is not correlated, so every element of $\hat{\boldsymbol{a}}^{\prime}$ can be searched independently. But $\hat{\boldsymbol{a}}^{\prime}$ has lost the integer property, the integer point corresponding to $\hat{\boldsymbol{a}}$ is difficult to confirmed. So we will determine the searching space of $\hat{\boldsymbol{a}}^{\prime}$ by the statistic parameter firstly. Then the searching space of $\hat{\boldsymbol{a}}$ can be obtained by $\boldsymbol{L}$ transformation.

The mathematical expectation of $\hat{a}: \boldsymbol{E}(\hat{\boldsymbol{a}})=\boldsymbol{a}$ based on the assumption that carrier phase double differenc observation obey positive distribution[11]. a is the truth value of ambiguity which can be transformed as $\boldsymbol{a}^{\prime}=\boldsymbol{L} \bullet \boldsymbol{a}$. The elements of $\hat{\boldsymbol{a}}^{\prime}$ are incorrelate, so the elements of $\hat{\boldsymbol{a}}^{\prime}$ are independent. So the element of $\hat{\boldsymbol{a}}^{\prime}: \hat{\boldsymbol{a}}^{\prime i}$ obey positive distribution with the mathematical expectation is $\hat{\boldsymbol{a}}^{\prime i}$. The confidence interval of $\hat{a}^{\prime i}$ wih confidence of $99.7 \%$ is $\left[a^{\prime i}-3, a^{\prime i}+3\right]$. On the other side, $a^{\prime i}$ can be seen as a random variable obeying positive distribution with the mathematical expectation is $\hat{a}^{\prime i}$. So the confidence interval of $a^{\prime i}$ wih confidence of $99.7 \%$ is $\left[\hat{a}^{\prime i}-3, \hat{a}^{\prime i}+3\right]$. Confidence interval of a can be write as

$$
\begin{aligned}
& \left\{\begin{array}{l}
\hat{a}^{1}-\frac{3}{l_{11}}<a^{1}<\hat{a}^{1}+\frac{3}{l_{11}} \\
\frac{C_{(i)}-3}{l_{(i)(i)}}+\hat{a}^{(i)} \leq a^{(i)} \leq \frac{C_{(i-1)}+3}{l_{(i)(i)}}+\hat{a}^{(i)}
\end{array}\right. \\
& \quad \text { And } C_{(i)} \text { is write as } \\
& C_{(i)}=\sum_{j=1}^{i-1} l_{i j}\left(\hat{a}^{j}-a^{j}\right), \quad 2 \leq i \leq n-1
\end{aligned}
$$

\section{EXPERIMENTAL RESULTS AND CONCLUSION}

On 10 January 2011, a static orientation test was performed employed two antennae and receivers made by Novatel Company. The frequency of sampling was set to be $1 \mathrm{~Hz}$. The antennae were fixed on the top of laboratory building with the length of baseline is $4.449 \mathrm{~m}$. Data were collected for almost 100 minutes while the number of visible satellites were 9 to ten. 6000 epochs were employed. The dominant frequency of the computer is $2.2 \mathrm{GHz}$, and the internal memory is $2 \mathrm{G}$. The performance index is showen in Table 1 .

MCT is meaning computing time; MAC is meaning ambiguity candidates; QSDEQ is quadratic sum of diagonal elements of $\boldsymbol{Q}_{\hat{\boldsymbol{a}}}$. It can be seen from table 1 that the constrainted model achieved higher computing rate, less number of ambiguities candidates, and smaller diagonal elements quadratic sum of variance-covariance of $\hat{\boldsymbol{a}}$. It means the method introduced in this contribution has higher computing efficiency.

\section{REFERENCES}

[1] Landan H, Euler H J, On-the-fly ambiguity resolution for precise differential positioning[A]. Processing of ION GPS-92 [C]. Albuquerque, New Mexico: 1992. 607-613. 
[2] ZHENG Qing-hui, ZHANG Yu-lin. OTF ambiguity resolution in GPS based attitued determination [J], Acta Aeronautica et Astronautica Sinica, 2002.23(3): 272-275.

[3] M. Coard, A. Geiger. Systematic, search for all possible widelanes[C]. Proceedings of the sixth international geodetic Symposium on satellite positioning. 312 318.1992.
[4] P. J. G. Teunissen. Least-squares estimation of the integer GPS ambiguities [C]. IAG General Meeting,Invited lecture, Section IV Theory and Methodology. Beijing, China, August, 1993.

[5] E.Frei, G.Beutler. Rapid static positioning based on the fast ambiguity resolution approach "FARA": theory and first results [J]. Manuscripta Geodaetica, 1990, 15 (4): 325-356.

\begin{tabular}{llll}
\multicolumn{4}{c}{ TABLE 1. Property OF CALCULATION } \\
\hline & MCT & MAC & QSDEQ \\
& & & \\
\hline Constrainted model & $1.51 \mathrm{~ms}$ & 331 & 24.24 \\
Unconstrainted model & $9.61 \mathrm{~ms}$ & 4282 & 36.35 \\
\hline
\end{tabular}

